\title{
DESARROLLO DEL BALANCED SCORECARD PARA LOGRAR LA CALIDAD EN LA FACULTAD DE CIENCIAS ADMINISTRATIVAS DE LA UNIVERSIDAD NACIONAL MAYOR DE SAN MARCOS
}

\author{
DEVELOPMENT OF THE BALANCED SCORECARD TO ACHIEVE QUALITY IN \\ THE FACULTY OF ADMINISTRATIVE SCIENCES, NATIONAL UNIVERSITY OF SAN MARCOS \\ Kennedy Narciso Gómez*** \\ kennedy_narc1@hotmail.com
}

[RECEPCIÓN: MARZO DE 2015 / CONFORMIDAD: ABRIL DE 2015]

\begin{abstract}
RESUMEN
El Balance Scorecard (BSC) de Kaplan y Norton, adaptado a organizaciones sin fines de lucro como herramienta de gestión pública, posibilitará mejorar la calidad de la Facultad de Ciencias Administrativas. Este instrumento guardará relación con el "Plan Bicentenario - Perú hacia el 2021", inserto en el Plan Estratégico Institucional 2012-2021 de la UNMSM, relacionado con el plan estratégico de la Facultad de Ciencias Administrativas 2012-2016, y el Modelo de Gestión ACBSP (Accreditation Council for Business Schools and Programs). El objetivo del BSC de la Facultad de Ciencias Administrativas de la UNMSM es lograr una adecuada gestión en la búsqueda de la calidad académica e institucional para entregar a la sociedad egresados bien educados. En una primera etapa, desarrollamos el diagnóstico de las oportunidades y necesidades de la Facultad de Ciencias Administrativas, contando para ello con el apoyo de alumnos, docentes, autoridades, y analizamos el direccionamiento de la misión, visión y valores indicados en el Plan Estratégico. En una segunda etapa procedimos a elaborar el modelo del BSC definiendo la visión, misión, objetivos, políticas y las cuatro perspectivas: 1 . Aprendizaje y conocimiento, 2. Proceso interno, 3. Cliente, 4. Financieras. En la tercera etapa elaboramos la matriz de riesgos y el programa de inversión económica del proyecto. Finalmente, consideramos implementar la propuesta de un software de manejo de indicadores del BSC en la Facultad de Ciencias Administrativas de la UNMSM, adaptado a organizaciones públicas como herramienta de gestión estratégica, que posibilitará la competitividad de la Facultad de Ciencias Administrativas de la UNMSM y lograr el éxito de su estrategia para alcanzar su misión.
\end{abstract}

Palabras clave: Gestión, perspectiva financiera, perspectiva de cliente, perspectiva de proceso interno, perspectiva de conocimiento y aprendizaje. Doctor en Administración. Magíster en administración. Bachiller en administración. Licenciado en Ciencias Administrativas.
Docente Auxiliar e Investigador del Instituto de Investigación de la Facultad de Ciencias Administrativas de la UNMSM. 


\begin{abstract}
The Balance Scorecard (BSC), adapted to nonprofit organizations Kaplan and Norton as public management tool that will allow improving the quality of the faculty of Administrative Sciences consistent with the "Bicentennial Plan - Peru 2021" insert in the Plan Institutional Strategy 2012-2021 of San Marcos related to the strategic plan of the Faculty of Administrative Sciences 2012- 016, and the Management Model ACBSP (Accreditation Council for Business Schools and Programs), which relate to our proposal of Balance Scorecard, which objective is to achieve management in the pursuit of academic and institutional quality and deliver to society well educated graduates. In a first step, we developed the diagnosis of opportunities and needs of the Faculty of Administrative Sciences, supported by students, teachers, authorities and analyze the routing of the mission, vision and values identified in the Strategic Plan; In a second step we proceeded to develop the model of Balance Scorecard (BSC), defining the vision, mission, objectives, policies and the four perspectives: (1) learning and knowledge, (2) internal process, (3) Customer) (4) Financial; In the third stage elaborate the risk matrix and economic investment program project. Finally, consider implementing a proposed management software BSC indicators in the Faculty of Administrative Sciences of San Marcos, adapted to public organizations Kaplan and Norton as a strategic management tool, which will enable the competitiveness of the FCA-UNAC and achieve the success of its strategy to achieve its mission.
\end{abstract}

Keywords: Management, financial perspective, customer perspective, internal process perspective, knowledge and learning perspective.

\section{INTRODUCCIÓN}

La Universidad Nacional Mayor de San Marcos, liderada por el Dr. Pedro Cotillo Zegarra (Rector período 2012-20l7), tiene la visión de integrar a la universidad a nivel del mundo en el marco de la globalización e internacionalización con las mejores universidades; asimismo, en el Plan Estratégico Institucional se plantean tres ejes estratégicos fundamentados en la educación superior, los cuales son: Educación de calidad e internacionalización; Investigación para el desarrollo humano sostenible; y formación humanística y creación cultural.

La Facultad de Ciencias Administrativas tiene su origen en el año 1875, cuando la Misión Francesa FODERÉ, al mando del jurista francés Pablo Pradier Foderé, crea la Facultad de Ciencias Políticas y Administrativas de la Universidad Nacional Mayor de San Marcos como parte del proceso de modernización del Estado en el gobierno de Don Manuel Pardo (1872-1876). En 1928, con la aprobación del nuevo Estatuto (Ley No 6041), la Facultad de Ciencias Económicas reemplazó a la anterior Facultad de Ciencias Políticas y Administrativas. Se transformó así mediante Ley $\mathrm{N}^{\circ} 7083$ del 9 de abril de 1931, siendo su primer decano don Abraham Rodríguez Dulanto, quien inició la consolidación de la Facultad a partir del inicio de sus actividades en el cargo, el 28 de mayo de 1931. En 1943 se crea la Facultad de Ciencias Económicas y Comerciales. En 1966, como consecuencia de las tendencias de aquel tiempo se crean los Programas de Contabilidad y Administración. En
1980 se produce la separación de los Programas y Departamentos de Contabilidad y Administración Es así como la Facultad de Ciencias Administrativas de la Universidad Nacional Mayor de San Marcos se creó en 1984, según Resolución Rectoral $N^{\circ}$ 78337, con su Escuela Académica Profesional de Administración (EAPA). A partir de su creación y gracias a la labor realizada por diferentes decanos, se logró materializar el Plan Estratégico 2012-2016 de la Facultad de Ciencias Administrativas, mérito del liderazgo de la decana Dra. Elizabeth Canales Aybar, importante para el tema de la investigación del BSC en la Facultad de Ciencias Administrativas para desarrollar un sistema de seguimiento y el control del cumplimiento de los objetivos estratégicos y su misión, que busca brindar una educación superior de alto nivel, entregando el valor intangible de la formación profesional y, a través de éstas, crear ciencia y tecnología.

El BSC se implementó en la Real Policía Montada de Canadá (RCMP) que es la organización policial histórica del país. Creada en 1873 e inspirada en la Royal Irish Constabulary (Guardia Civil Real Irlandesa) y las unidades montadas del ejército de Estados Unidos, la Real Policía Montada del Noroeste fue creada para llevar la ley, el orden y el gobierno canadiense a los territorios del noroeste (hoy en día Alberta y Saskatatchewan). Actualmente, la Real Policía Montada de Canadá es el servicio nacional del policía canadiense y una dependencia del Ministerio del Procurador General de Canadá. La RCMP es única en el mundo dado que es, simultáneamente, un cuerpo de 
policía nacional, federal, provincial y municipal. El mapa estratégico de la RCMP fue adoptar el BSC como herramienta para gestionar la ejecución del marco estratégico. EL BSC le permitió lograr lo siguiente: traducir el marco estratégico en una serie de objetivos, indicadores y metas coherentes; que la responsabilidad por los resultados del marco estratégico fuera más abierta y transparente; asegurar la alineación organizacional desdoblando el BSC a cada una de las divisiones y áreas funcionales de la organización.

La Facultad de Ciencias Administrativas de la UNMSM requiere construir un sistema de medición que describa la estrategia, ese modelo para nosotros se llamará el Cuadro de Mando Integral; asimismo, fija los siguientes objetivos del Balanced Scorecard en la FCA-UNMSM: diseñar y desarrollar la visión, misión, políticas; desarrollar las perspectivas de los aprendizajes y conocimientos; desarrollar las perspectivas del proceso interno; desarrollar las perspectivas del cliente; y desarrollar las perspectivas financieras.

El presente trabajo de investigación presenta el siguiente problema:

¿Cómo influye el Balanced Scorecard en la calidad de la Facultad de Ciencias Administrativas de la UNMSM?

\section{MÉTODOS}

Diseño de investigación

\begin{tabular}{|c|c|c|c|}
\hline UNIDAD DE ANÁLISIS & INFORMACIÓN NECESARIA & UNIDAD DE OBSERVACIÓN & TÉCNICA \\
\hline $\begin{array}{l}\text { 1. Documentos oficiales (plan Institucional, } \\
\text { Plan Operativo, Programa ACBSP (Accre- } \\
\text { ditation council for Businnes Schools And } \\
\text { Programs) Plan Estratégico de la FCA, } \\
\text { memoria anual 2013, 2014, estatuto, Ley } \\
\text { Universitaria, plan bicentenario nacional } \\
\text { y su relación con el plan estratégico de la } \\
\text { FCA-UNMSM. } \\
\text { 1.1. Diagnóstico macro y micro ambiente }\end{array}$ & $\begin{array}{l}\text { 1. Cuándo, dónde, quiénes y cómo se } \\
\text { relaciona la estrategia con la herra- } \\
\text { mienta del BSC } \\
\text { 1.1. Qué dificultades se encuentran, } \\
\text { cuánto es el costo de trabajar sin el } \\
\text { proyecto del BSC y con el proyecto } \\
\text { del BSC. } \\
\text { 1.2. Utilizan asesores externos. } \\
\text { 1.3. Informe relevante técnico del } \\
\text { diagnóstico. }\end{array}$ & $\begin{array}{l}\text { 1. Servidores público no docentes } \\
\text { (interno, externo) } \\
\text { 1.1. Servidores publicos docentes. } \\
\text { 1.2. Directivos de áreas } \\
\text { 1.3. Vicerrector de Investigación, } \\
\text { - Vicerrector académico } \\
\text { 1.4. Estudiantes } \\
\text { 2. Rector. } \\
\text { 2.1. Acervo documental de informe de } \\
\text { diagnóstico técnico. }\end{array}$ & $\begin{array}{l}\text { 1. Muestra aleatoria } \\
\text { 1.1. Docentes, autorida- } \\
\text { des, Estudiantes. }\end{array}$ \\
\hline $\begin{array}{l}\text { 2. Elaboración del Balance Scorecard de la } \\
\text { FCA-UNMSM. 2014-2021. }\end{array}$ & $\begin{array}{l}\text { - Tecnologías disponibles. } \\
\text { - Modelo BSC-FCA-UNMSM. } \\
\text { - Proceso de las fases de Formula- } \\
\text { ción, Implementación, Control. }\end{array}$ & & \\
\hline
\end{tabular}

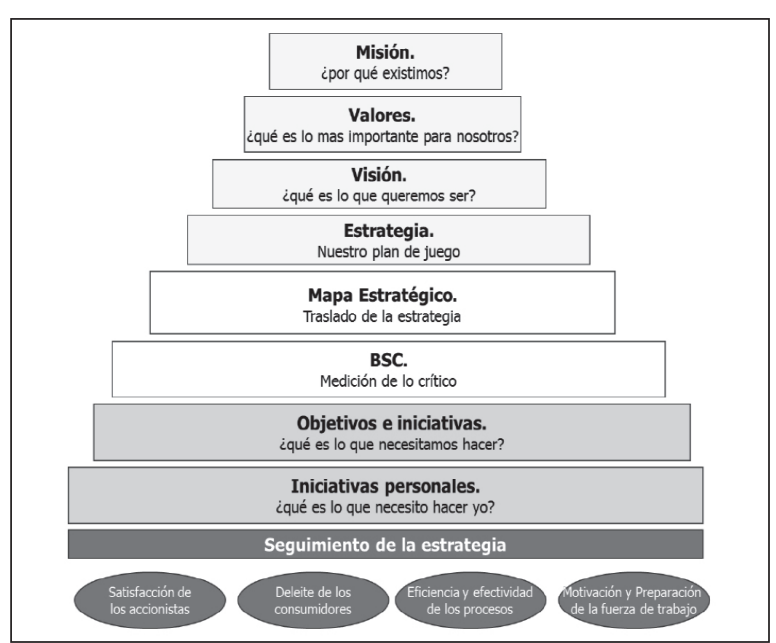

Fig. $\mathrm{N}^{\mathrm{o}} 1$.

El Balanced Scorecard en organizaciones sin fines de lucro ${ }^{1}$ Fuente: Kaplan y Norton 2001.

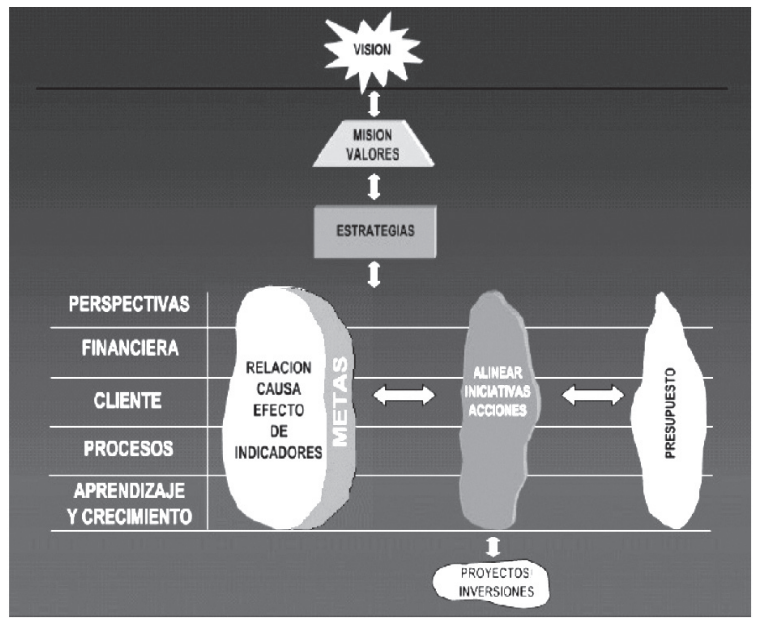

Fig. $\mathrm{N}^{\mathrm{o}} 2$.

El Balanced Scorecard en organizaciones sin fines de lucro Fuente: Kaplan y Norton 2001.

1 Kaplan y Norton Mapas Estratégicos Gestión 2000 Barcelona 2001 


\section{RESULTADOS}

1. El cuadro de mando integral una propuesta para la Universidad Nacional Mayor de San Marcos.

La Universidad Nacional Mayor de San Marcos cuenta con el Plan Estratégico Institucional 2012-2021, aprobado según Resolución Rectoral No 01470-R-12 de fecha 15 de marzo del 2012; sin embargo, no cuenta con la herramienta de gestión denominada Balanced Scorecard (BSC), pues, organizaciones de alto desempeño la utilizan mediante una combinación de indicadores financieros y no financieros para aumentar el involucramiento, establecer equipo e impulsar a la organización a la competitividad. El BSC le permitirá lograr lo siguiente: traducir el marco estratégico en una serie de objetivos, indicadores y metas coherentes; en otras palabras, el BSC describiría y mediría la estrategia de la universidad; lograr que la responsabilidad por los resultados del marco estratégico sea más abierta y transparente; y, asegurar la alineación organizacional desdoblando el BSC a cada una de las facultades académicas y áreas funcionales de la universidad.
1.1 Mapa Estratégico de la Facultad de Ciencias Administrativas de la Universidad Nacional Mayor de San Marcos.

El equipo organizó su mapa estratégico luego de esfuerzos seguido en tres momentos: 1. Reuniones con docentes y estudiantes generosos; 2 . Conocer la realidad y necesidad nacional e internacional, cuyo propósito es diferenciarnos de las demás universidades; 3 . Sociedad, atrayendo a una nueva generación de mejores alumnos de los colegios nacionales e internacionales y con una formación académica adecuada para devolverlos a la sociedad como egresados bien educados. Así, Narciso Gómez dice lo siguiente: "En mi experiencia como pas-decano de la Facultad de Ciencias Administrativas de la Universidad Nacional del Callao en dos periodos (2005 y 2010-2013) y como director general de la Oficina Central de Cooperación Técnica Internacional de la UNAC, pude apreciar que los egresados, alumnos de alto nivel académico de los colegios prefieren postular a universidades privadas. Presentamos el resumen del mapa estratégico y apreciamos las relacionas causales de los objetivos con las estrategias identificadas, desde las diferentes perspectivas". Véase la Figura 3.

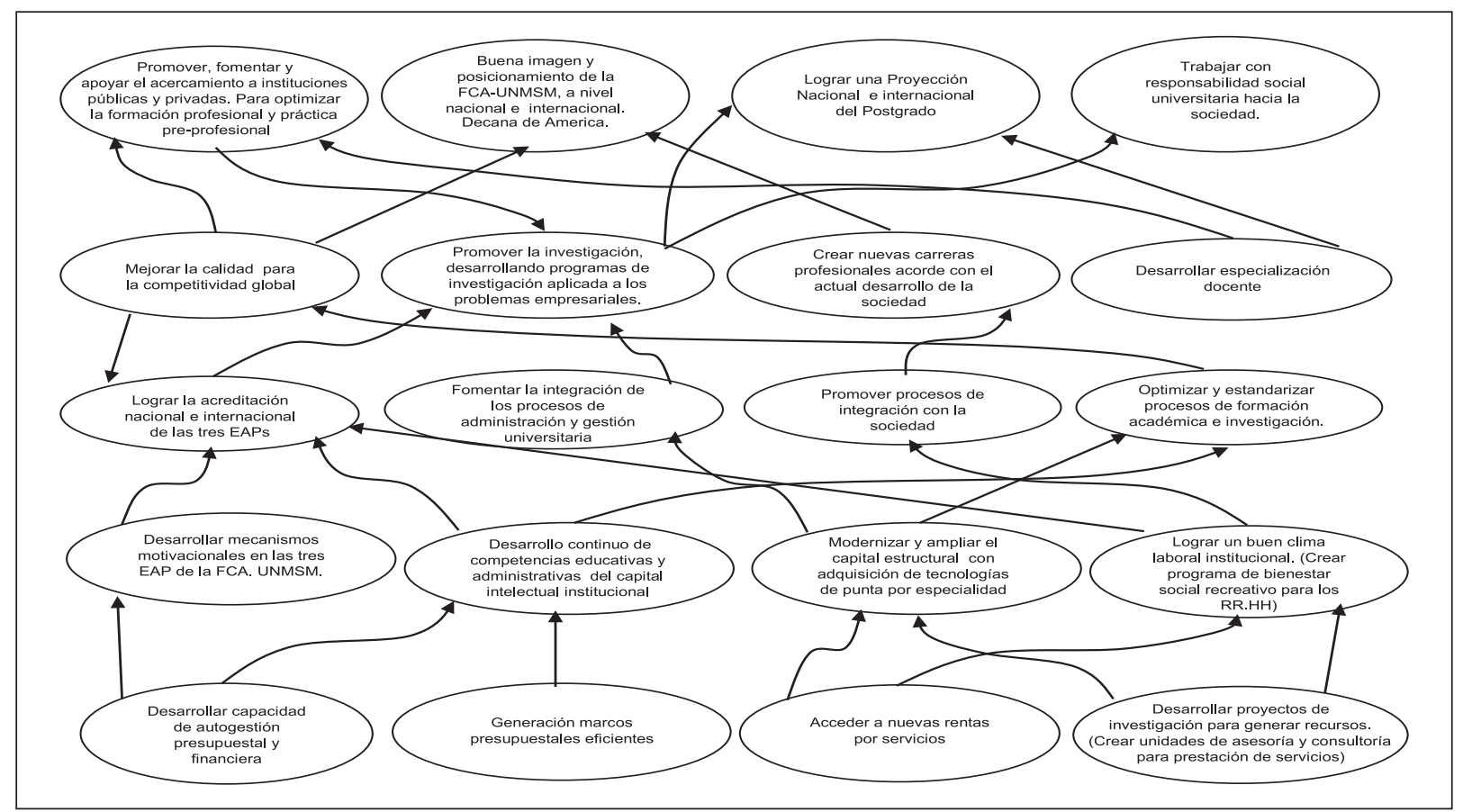

Fig. $\mathrm{N}^{\mathrm{o}} 3$

Mapa Estratégico de la Facultad de Ciencias Administrativas

Fuente: Elaboración Propia. 


\subsection{Modelo de enlace de los objetivos estratégicos en el cuadro de mando integral a usar en la FCA de la UNMSM.}

La Facultad de Ciencias Administrativas muestra los objetivos que le permitirán ayudar a cumplir con su misión de manera integral. Véase Figura No 4 y Figura № 5.

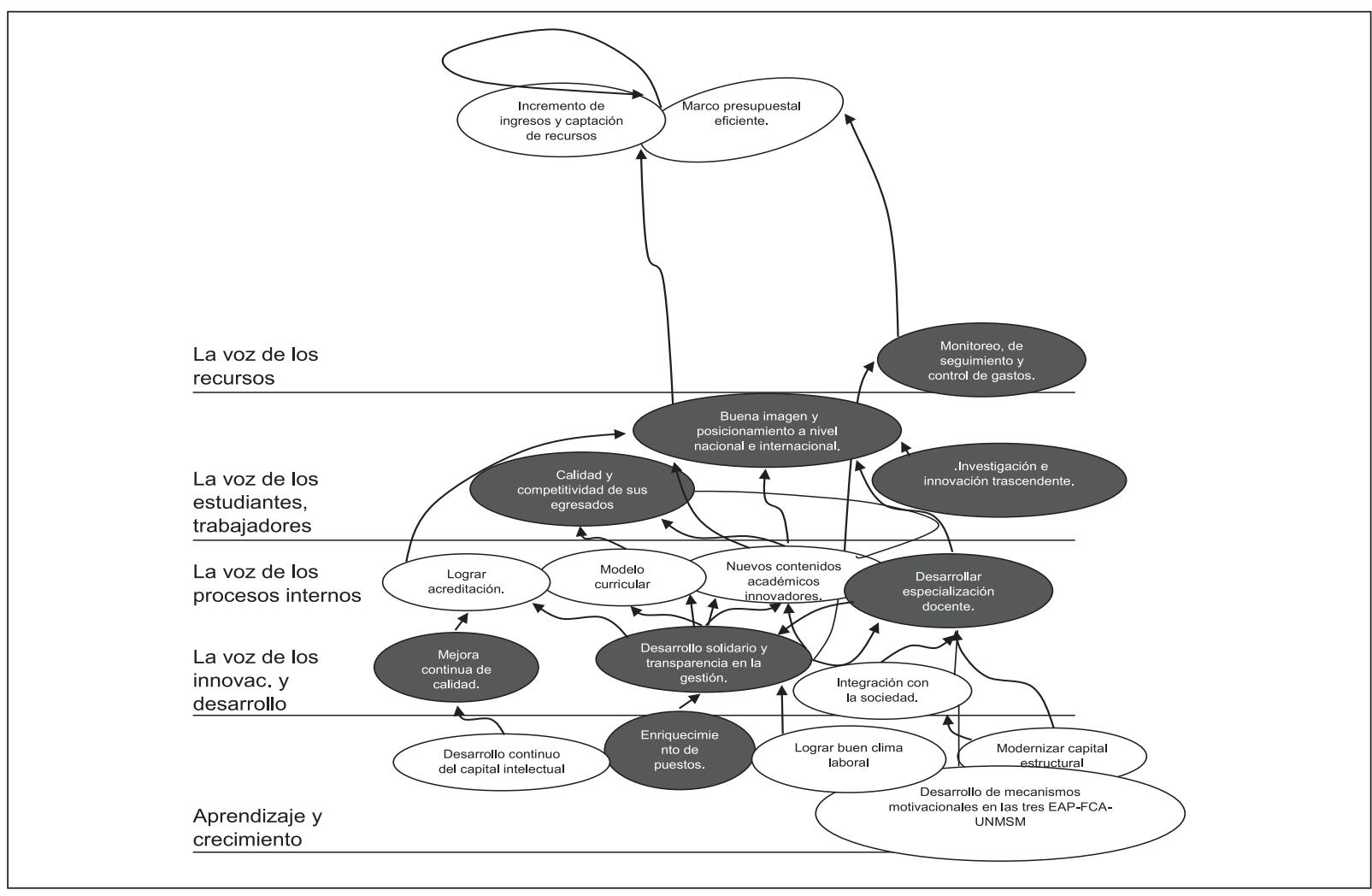

Fig. $\mathrm{N}^{\mathrm{O}} 4$.

Enlace de los objetivos estratégicos en el cuadro de mando integral.

Fuente: Elaboración propia.

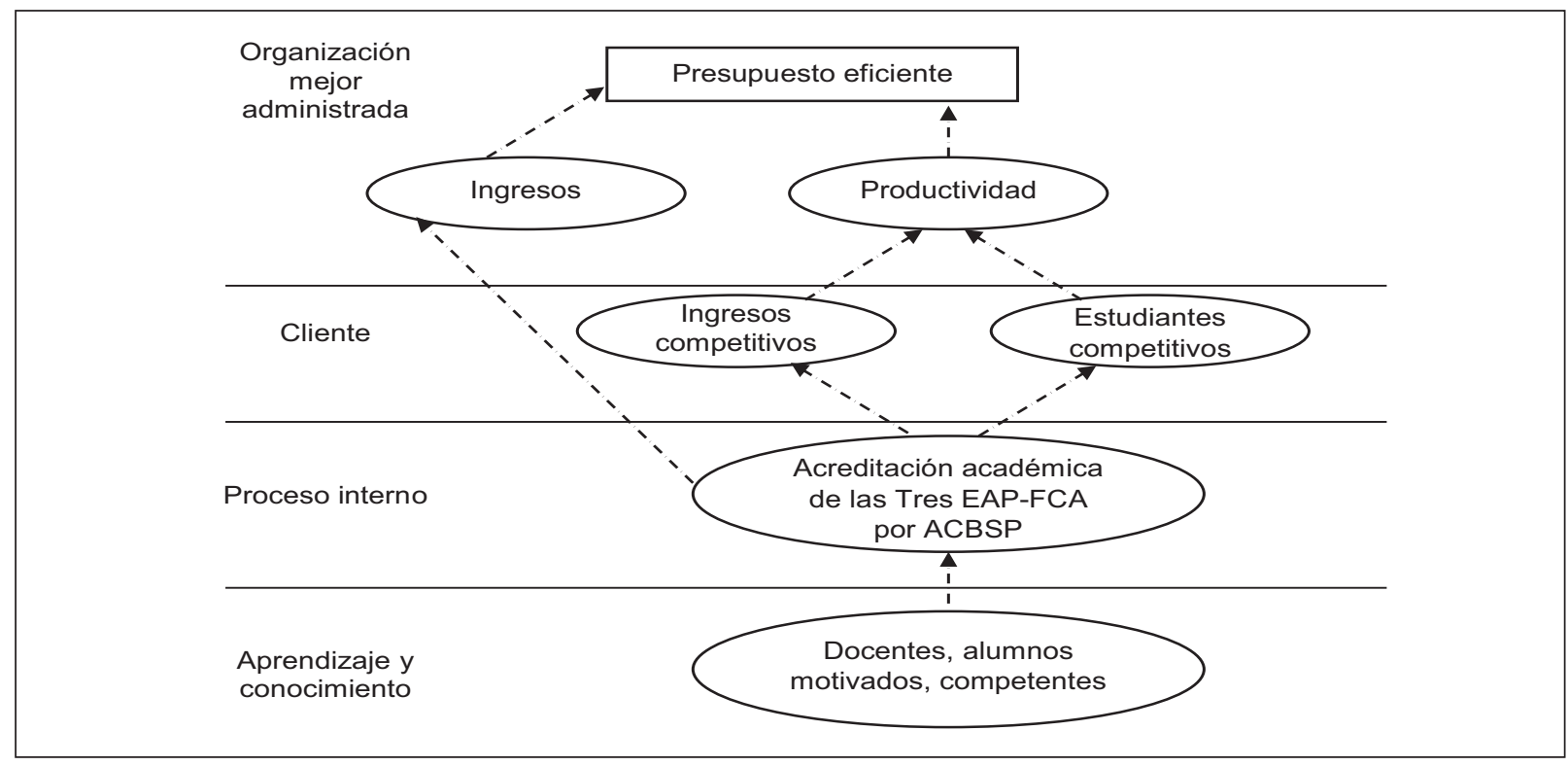

Fig. $\mathrm{N}^{\mathrm{o}} 5$.

Balance Scorecard de la FCA-UNMSM

Fuente: Elaboración Propia. 


\subsubsection{Visión y misión de la Facultad de Ciencias Administrativas de la UNMSM.}

Visión

Ser reconocida como la facultad de excelencia, líder internacional en generación y transmisión de conocimientos en ciencias administrativas y en la formación de profesionales con actitud emprendedora en un contexto global y de responsabilidad social.

\section{Misión}

Somos la Facultad dedicada a la formación y perfeccionamiento de profesionales en ciencias administrativas, comprometidos con la mejora continua y la práctica de valores que contribuyen con el desarrollo sostenible del país.

\subsubsection{Lineamientos de políticas de la FCA de la UNMSM²}

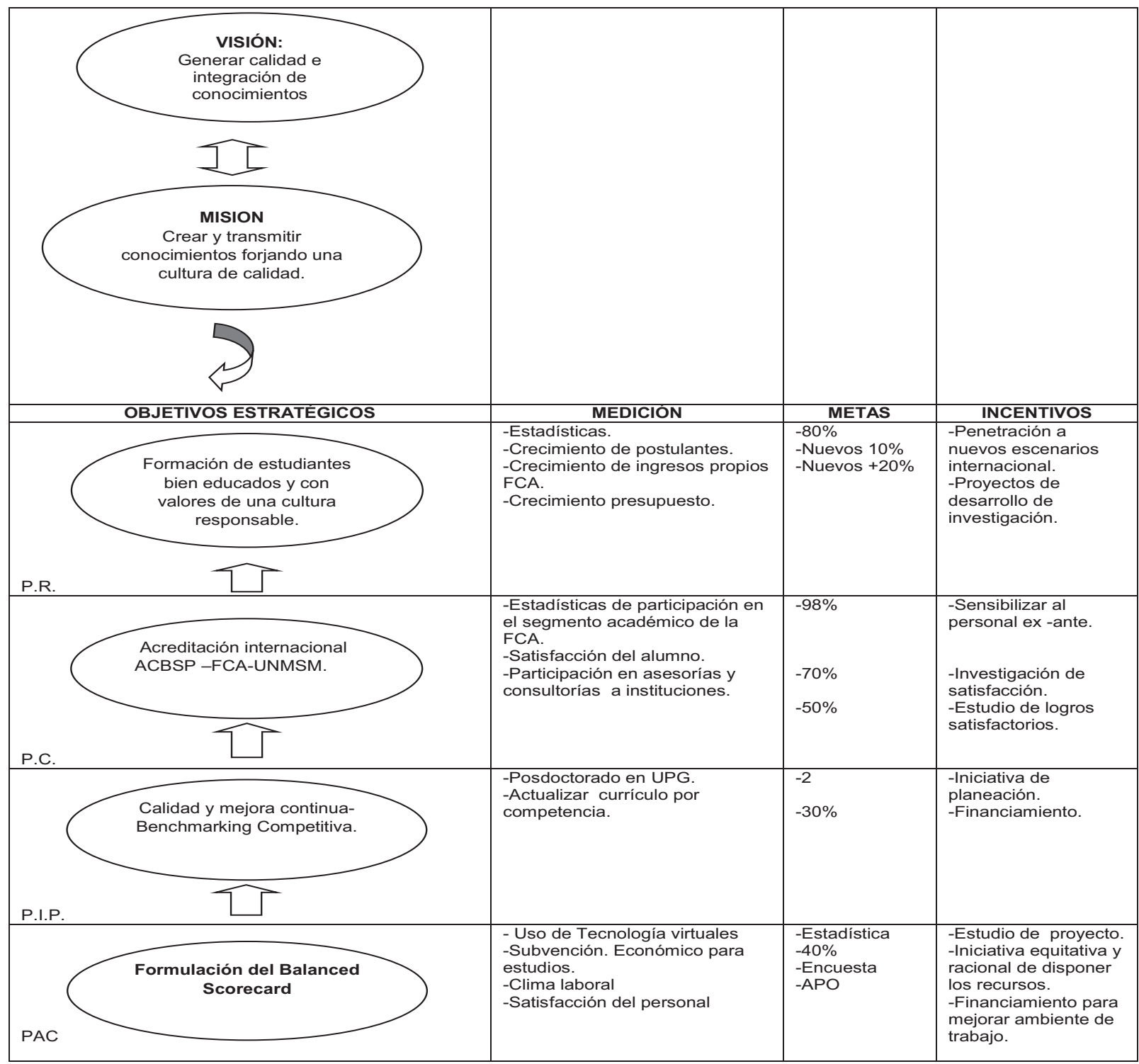

Fig. $\mathrm{N}^{\mathrm{o}} 6$.

Perspectivas en los proceso del BSC en la FCA-UNMSM.

Fuente: Elaboración Propia.

2. Plan Estratégico 2012-2016-FCA-UNMSM. 


\section{DISCUSIÓN}

El principio fundamental subyacente del BSC: es "Si se puede medir, se puede gestionar", el problema no es una mala estrategia sino una mala ejecución.

Esta práctica exige una conciencia y compromiso integral de las facultades y la universidad sobre la naturaleza del Balanced Scorecard que clasifica los procesos internos en cuatro perspectivas alineadas con cada objetivo. Cada objetivo es: Objetivo único (nuestro objetivo de la FCA es implementar el BSC para lograr la calidad académica, para ello tenemos que concretar acreditar a la FCA); Objetivo común (aumentar ingresos propios, a través de centro de producción, matrícula de alumnos, cursos de titulación y extensión universitaria, estudios de maestrías y doctorados, cursos de idiomas, etcétera; y Objetivo compartido: un objetivo obligatorio para la facultad (Lograr la calidad académica, requerida en la perspectiva de proceso internos del BSC de la FCA-UNMSM).

En esa misma dirección, la Facultad de Ciencias Administrativas tiene el reto de lograr dos objetivos fundamentales: la formación de profesionales, científicos e intelectuales y la realización de las investigaciones científicas para crear nuevos conocimientos para el desarrollo de la ciencia y tecnología de nuestro país.

\section{CONCLUSIONES}

1. La Facultad de Ciencias Administrativas debe implementar el Balanced Scorecard que permita gestionar la estrategia a lo largo de la facultad, a través de éste se crearán las medidas que le servirán para guiar las acciones de todos los empleados hacia el logro de la misión.

2. La implementación deberá tomar acciones para cumplir las promesas de valor. Ello implica: Primero, requerimos el compromiso del rector, de la decana y de los miembros del Consejo de Facultad, para que el Balanced Scorecard se implemente. Segundo, deberá ser aprobado el BSC con su presupuesto y el equipo conductor por el Consejo de Facultad de la FCA y ratificado por el Consejo Universitario. Tercero, cumplir con las presentaciones parciales se- mestrales, disertadas y superando las brechas que se suscitan. Cuarto, presentación de un informe anual sustentado ante las autoridades correspondientes y este proceso deberá ser de mejora continua.

3. El proceso del BSC exige cumplir cuatro perspectivas:

\subsection{Perspectiva de los resultados}

El BSC permitirá crear el valor en la formación de egresados bien educados, ubicados en organizaciones de prestigio.

Implica: Estudios de ampliación de nuevos escenarios en el ámbito nacional e internacional con perspectivas de generar valor único superior.

\subsection{Perspectiva del cliente}

- Usar ratios de productividad para conocer la productividad del trabajo académico administrativo: relación alumno docente, sueldo del docente por hora-hombre, número de horas académicas, número de horas administrativas del docente, número de investigadores con-con y sin-sin; número docentes con grado de doctor, magíster y post-doctoral.

- Buscar acercamiento a través de proyecto de modelos de transferencia de conocimientos académicos mediante la Oficina de Cooperación Técnica Internacional de la UNMSM con universidades de prestigio de países integradas a la Alianza Estratégica de Universidades del Perú.

- Estudio del grado de percepción de los clientes y no clientes acerca de la FCAUNMSM.

\subsection{Perspectiva interna}

- Creación de programa post doctorado en la UPG-FCA-UNMSM.

- Actualizar currículo de estudios en función a requerimientos de las organizaciones de la sociedad.

- Iniciativas de planes de financiamiento. 


\subsection{Perspectiva del aprendizaje}

- Actualizar la hoja de vida en la oficina de escalafón para conocer las competencias del personal: grados académicos (magíster, doctor, especialistas, etcétera), para proponer mejoras.

- Inversión en programas de estudios al personal.

- Gestión interna y externa de relaciones humanas.

\section{LITERATURA CITADA}

Kaplan, Robert y Norton, David. (2001). Mapas Estratégicos. Gestión 2000, Barcelona.

Universidad Nacional Mayor de San Marcos. (2012). "Plan estratégico de la Facultad de Ciencias Administrativas de la Universidad Nacional Mayor de San Marcos 2012-2016". En: Revista Facultad de Ciencias Administrativas. UNMSM, Lima. 